\title{
Emulation Based Services in Digital Preservation
}

\section{$J C D L 2010$}

21 - 25 June in Surfers Paradise Australia

Dirk von Suchodoletz, Klaus Rechert

Faculty of Engineering, University Freiburg 


\section{Enabling Access to Emulation}

- Emulation in digital preservation is an acknowledged method to

- Preserve a wide range of different obsolete computer platforms

- Offer access to complex, dynamic and interactive digital objects

- Revive complete digital ecosystems of a number of different hardware and software components 


\section{Enabling Access to Emulation}

- Emulation environments

- Often deal with outdated concepts of software interaction

- Typically complex and require specific knowledge

- Require depending on the digital object to be rendered or executed a bunch of additional software components which may need prior installation 


\section{Enabling Access to Emulation}

Albert-Ludwigs-Universităt Freiburg

- Major goal is to allow non-technical users access to those services an easy to use, abstract interface is required

- During PLANETS project we developed and demonstrated a prototype for emulation wrapping - GRATE

- Different emulators like Dioscuri, MESS, QEMU, Hatari and others put into a single networked application
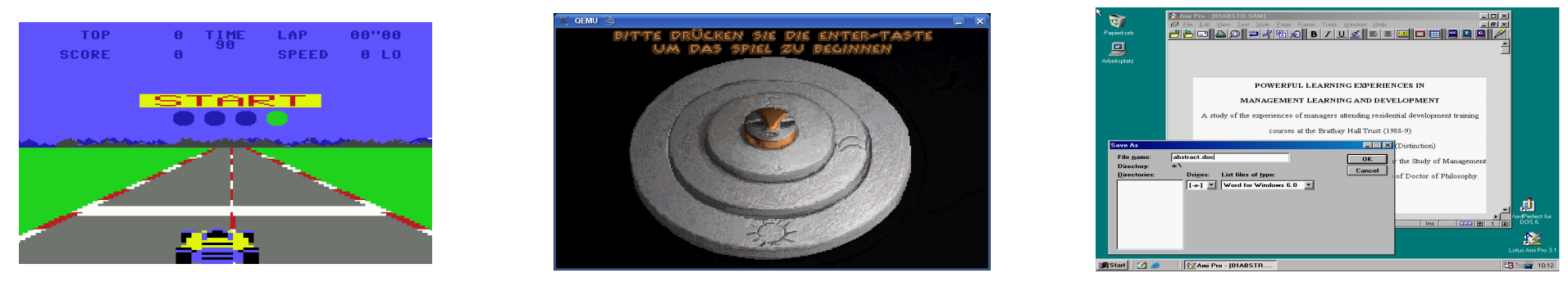


\section{Remote Emulation}

Albert-Ludwigs-Universität Freiburg

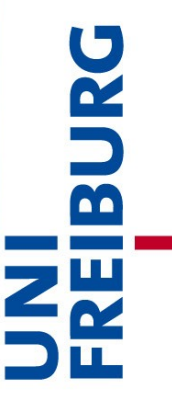

- Screen output and mouse+keyboard handled via event and transportation layer

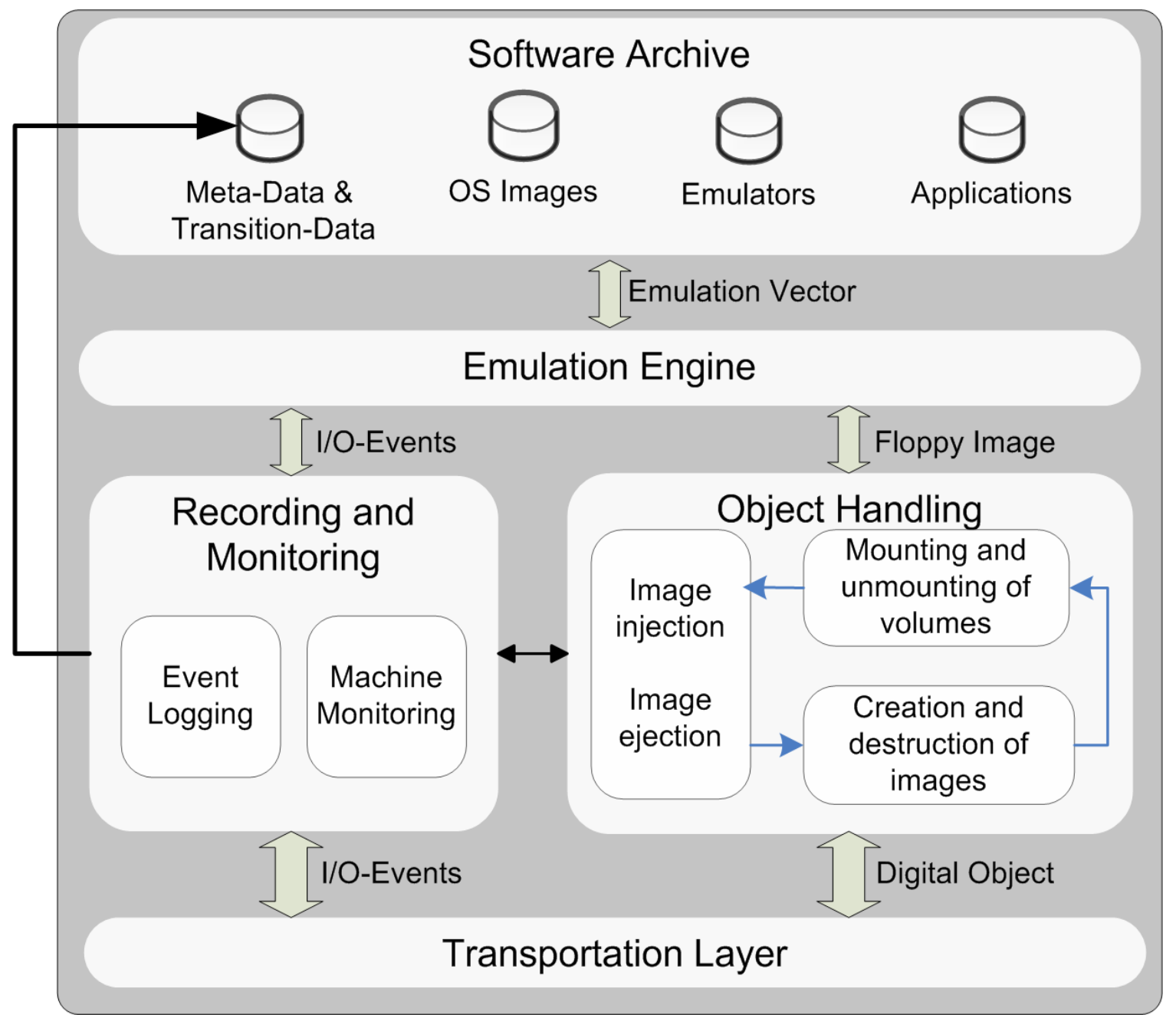
environment 


\section{Issues in Remote Emulation Services}

- Transportation - Attaching and retrieving digital objects to and from the runtime environment

- Software Archive - Most of the digital objects need further software components to be executed, rendered or viewed like applications, operating systems, drivers or components like fonts, codecs or special libraries

- Knowledge and automation - Preserving specific knowledge about an ancient environment and its applications 


\section{Object Transport}

Albert-Ludwigs-Universität Freiburg

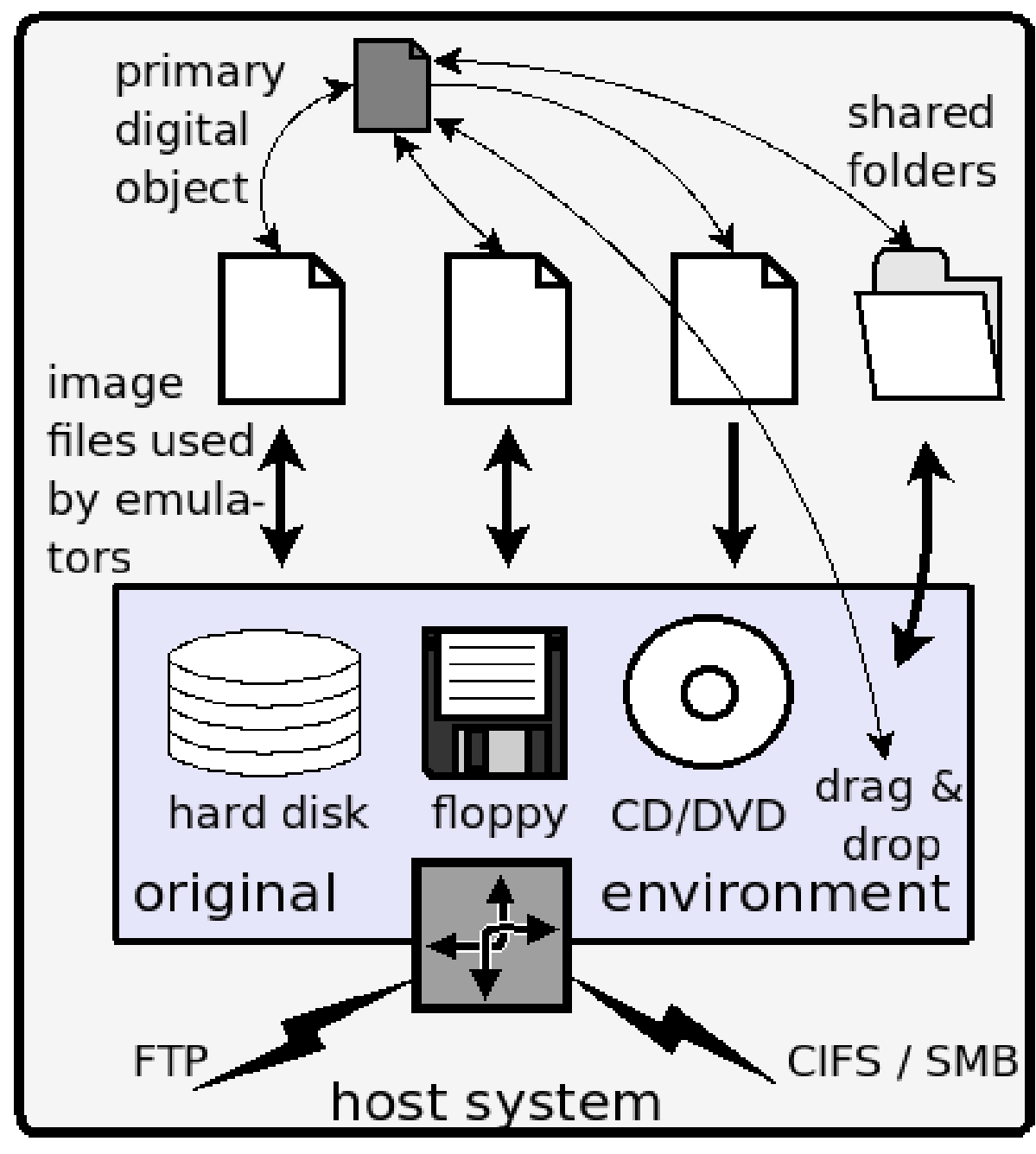

Digital Object needs

- Passed to remote emulation server

- Into the emulation environment

- Two distinct steps latter one depending on emulated system 


\section{Software Archiving}

Albert-Ludwigs-Universität Freiburg

- Digital objects not usable out of themselves suitable context is required (digital ecosystem)

- Software archive containing all necessary additional objects or prefabricated view-path

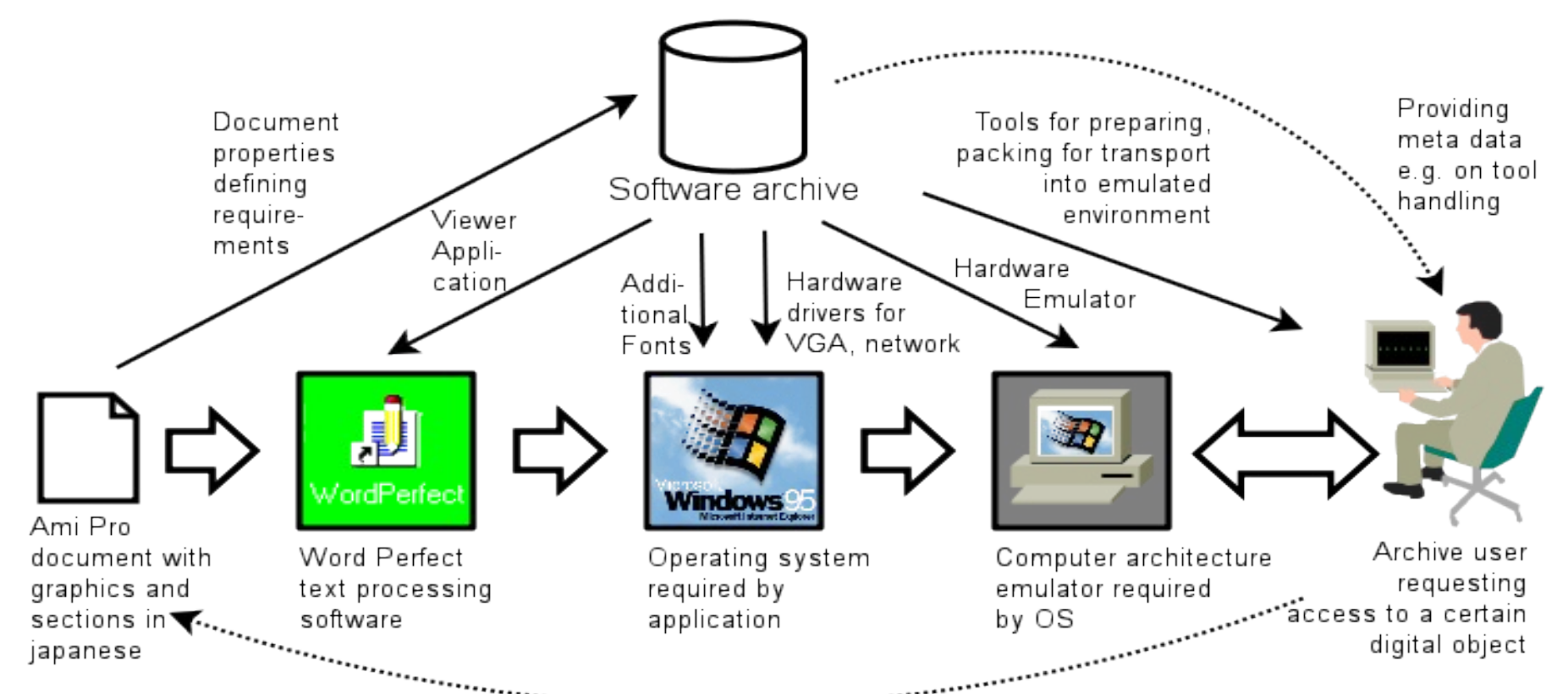




\section{Knowledge and Automation}

- GRATE focuses on traditional human interaction model, but

- Requires certain knowledge getting more and more uncommon for todays users

- Unsuitable for integration into noninteractive large scale preservation workflows 


\section{Knowledge and Automation}

Albert-Ludwigs-Universität Freiburg

- Typical applications most digital objects produced with are interactive

- Standard work-flows like opening a document and save it in a different format require a human user to type or point\&click

- $\quad$ Such manual procedure sub-optimal for e.g. mass migration scenarios

- We suggest a method to replace the humaninteraction in GRATE with generic recording and monitoring 


\section{Automation of Interaction}

Albert-Ludwigs-Universität Freiburg

- We see an interactive work-flow as ordered list of interactive events passed on (e.g. mouse and keyboard events)

- Each event is linked with a precondition and an expected outcome

- Built our solution on top of the VNC-Play tool, which offers visual synchronization points

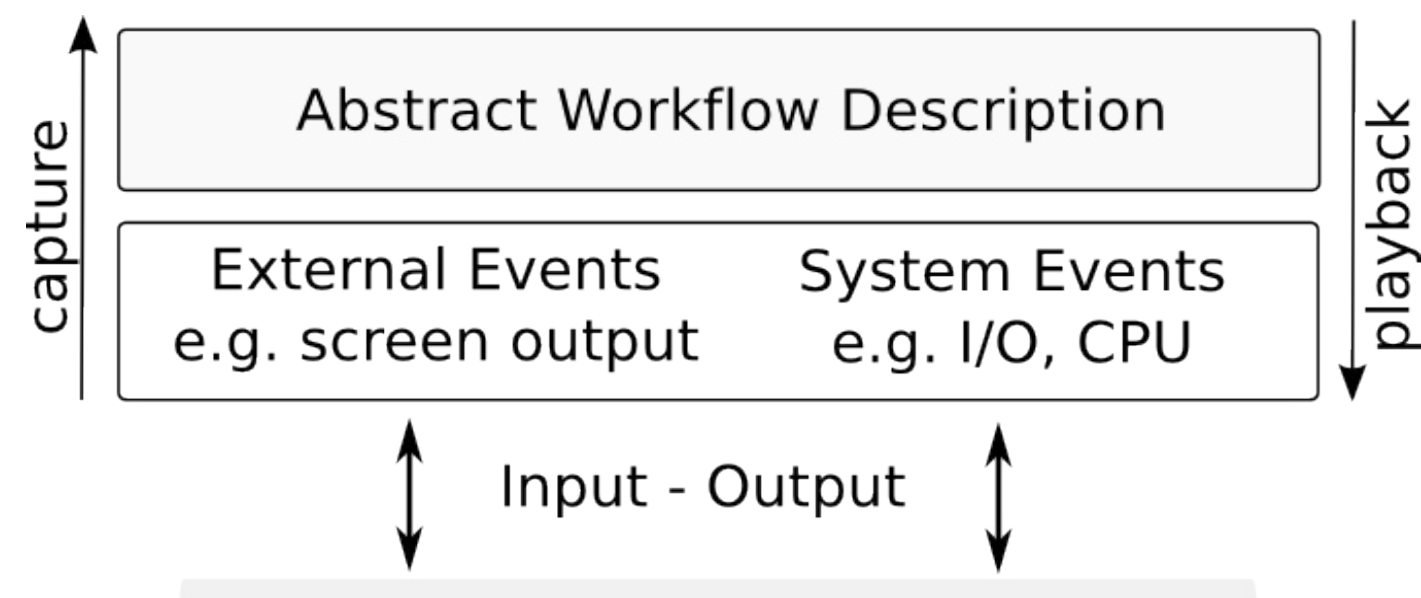

Input-Output Abstraction Layer

Emulated System Environment 


\section{Framework Integration}

Albert-Ludwigs-Universităt Freiburg

- PLANETS - Preservation and Longterm Access to NETworked Services

- Offers a set of standardized Web services like Characterization, View, Validation, Comparison, Migration, ...

- Defines a set of APIs Web services need to conform to 
- Emulation services should allow

- Occasional users to view digital objects and compare digital objects in their original environment

- Occasional users to experience ancient (graphical) interactive user environments

- Documentation and preservation of user interactions and interactive processes in ancient user environments

- Automated migration of files using the original application in emulation 


\section{Challenges}

- After reviewing these goals we see the need of

- View service to allow traditional interactive access to objects

- Automated migration by emulation service 


\section{Emulation View Service}

- A generic PLANETS view service takes a digital object and returns an URI pointing to the rendered result

- If the digital object requires a running rendering engine the service offers methods for querying and sending

- Our view service allows access to already configured and ready-made software environments 


\section{Emulation View Service}

- Implemented as a PLANETS Web service

- Accepts a list of digital objects

- Wraps them into a CD image

- Makes them available for running operating system

- User is able

- Explore the original environment

- Use within the original application

- Allows visual comparison for migrated objects

- Do manual migrations by saving or printing

- Process can be generalized, recorded 


\section{Emulation View Service}

Albert-Ludwigs-Universität Freiburg

\section{PLANETS Emulation View Service}

The PLANETS view Web service interface is designed to render a digital object. The service takes a digital object and returns an URI pointing to the rendered result. If the digital object requires a running rendering engine, the service offers methods for querying the engine's state and allows sending commands to it.

The emulation view service allows access to already configured and ready-made emulators and software images. The web service accepts a list of digital objects, wraps them into floppy or $\mathrm{CD}$-images and injects them into the running OS. The user is able to explore the environment, to use the digital objects with their original application and compare the result visually with their appearance in current applications or migrated versions of them.

Please select the appropriate Qemu image and inject files for manipulation(if any). Afterwards press "Start" button.

\section{Avaliable images:}

Windows 311.qcow

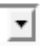

\section{File injection:}

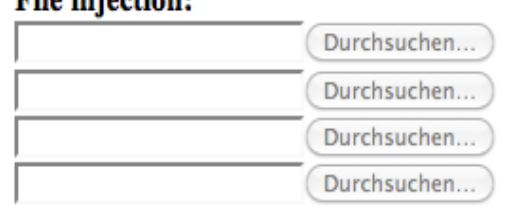

Start Stop Clear

\section{Disconnect Options Clipboard Send Ctrl-Alt-Del Refresh}

$\underline{\underline{D} a t e i} \underline{0 p}$ tionen $\underline{\text { Fenster }}$ Hilfe Programm-Manager

\begin{tabular}{|c|c|c|c|}
\hline - & Hauptg & uppe & 1. \\
\hline$\underbrace{}_{\text {Datei-Manager }}$ & Systemsteuerung & Druck-Manager & $\begin{array}{c}\text { Ablagemappe } \\
\text { Ablu }\end{array}$ \\
\hline $\begin{array}{c}\text { MS } \\
\text { MS.DOS. } \\
\text { Eingabeaufforderung }\end{array}$ & Windows-Setup & $\begin{array}{l}\text { PIF-Editor } \\
\text { PIIF }\end{array}$ & $\underset{\text { Read Me }}{\infty}$ \\
\hline
\end{tabular}

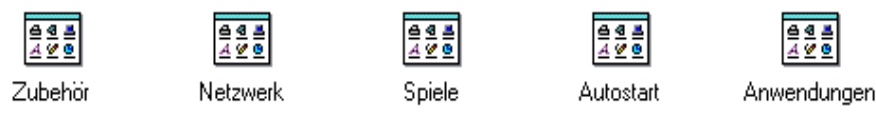

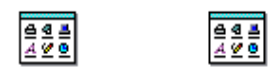

Lotus Applications HP LaserJet 


\section{Migration by Emulation Service}

- Good for viewing but not for large scale preservation tasks

- Second important service for PLANETS using emulation

- Interface expects a digital object as input, a designated output format (PUID) and an optional list of service parameters

- Outcome will be a successfully transformed object or an error message 


\section{Outlook: General Integration}

Albert-Ludwigs-Universität Freiburg

- Integrate software archive into preservation work-flows

- Check software list on object ingest

- Store single software components

- Documentation

- Preserve knowledge by storing work-flow recordings and complete emulation environments

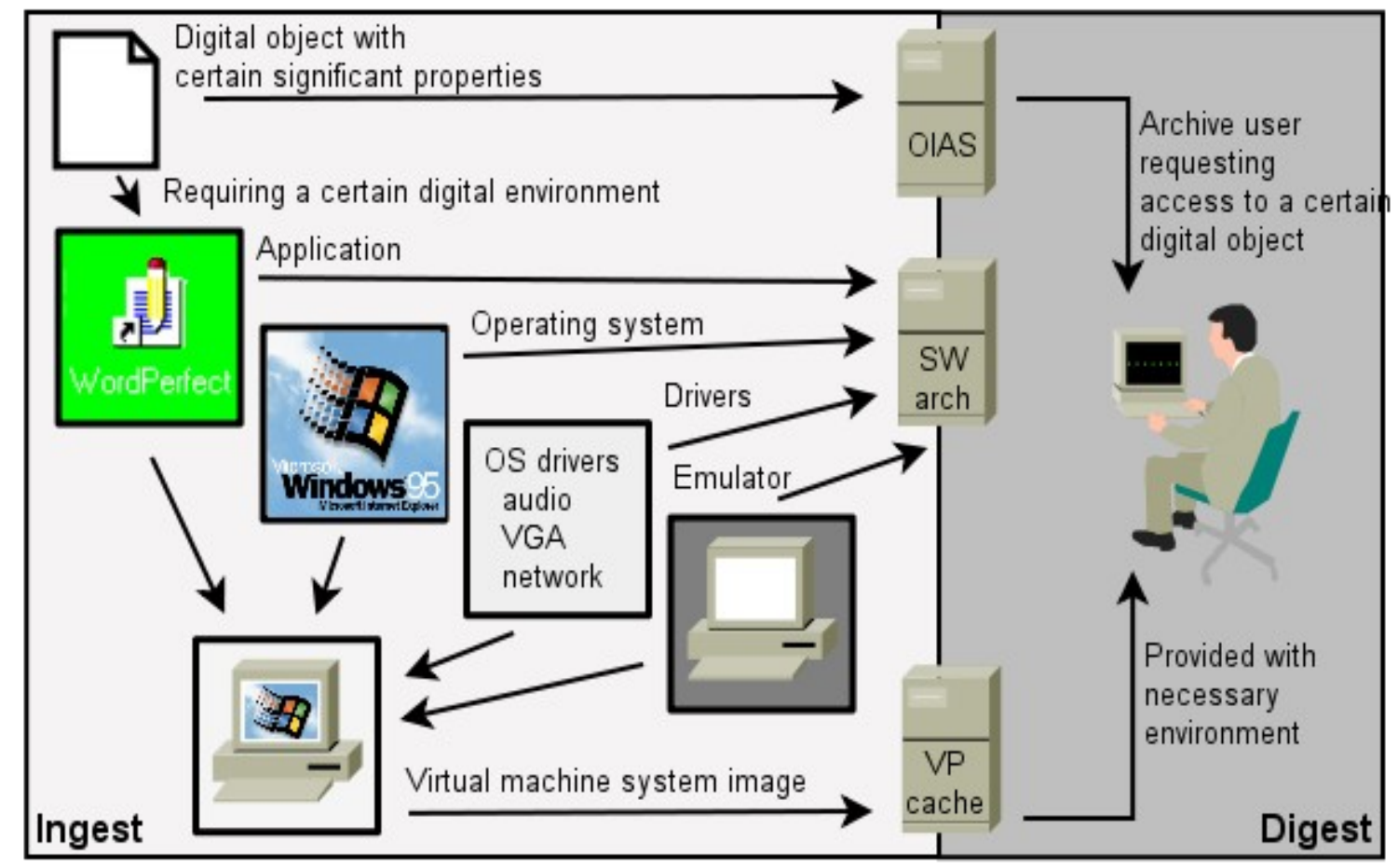




\section{Summary / Conclusion}

- Achievements

- Offer emulation to arbitrary, non-expert users

- Make emulation available to preservation frameworks

- Wrap emulation services via simple APIs

- Open research issues

- Make emulation more predictable and open to measurements 


\section{Thank you for your Attention!}

\section{Questions / Comments?}

Dirk von Suchodoletz Klaus Rechert

\section{plonets}

Chair on Communication Systems

Faculty of Engineering

University of Freiburg / Germany

klaus.rechert,dirk.von.suchodoletz@rz.uni-freiburg.de 


\section{Advantages of Remote Emulation}

Albert-Ludwigs-Universität Freiburg

- Avoids often sophisticated installation of a wide range of different software components

- No hassles with unpredictable environments on users side

- Avoids the transfer of proprietary software to the emulation users

- Could be implemented as a cloud service run by specialized memory institutions

- Might offer non-traditional ways to present digital collections e.g. computer games 